\title{
MLGrafViz: multilingual ontology visualization plug-in for Protégé
}

\author{
Merlin Florrence \\ Sacred Heart College, Tirupattur, Tamil Nadu 635601, India
}

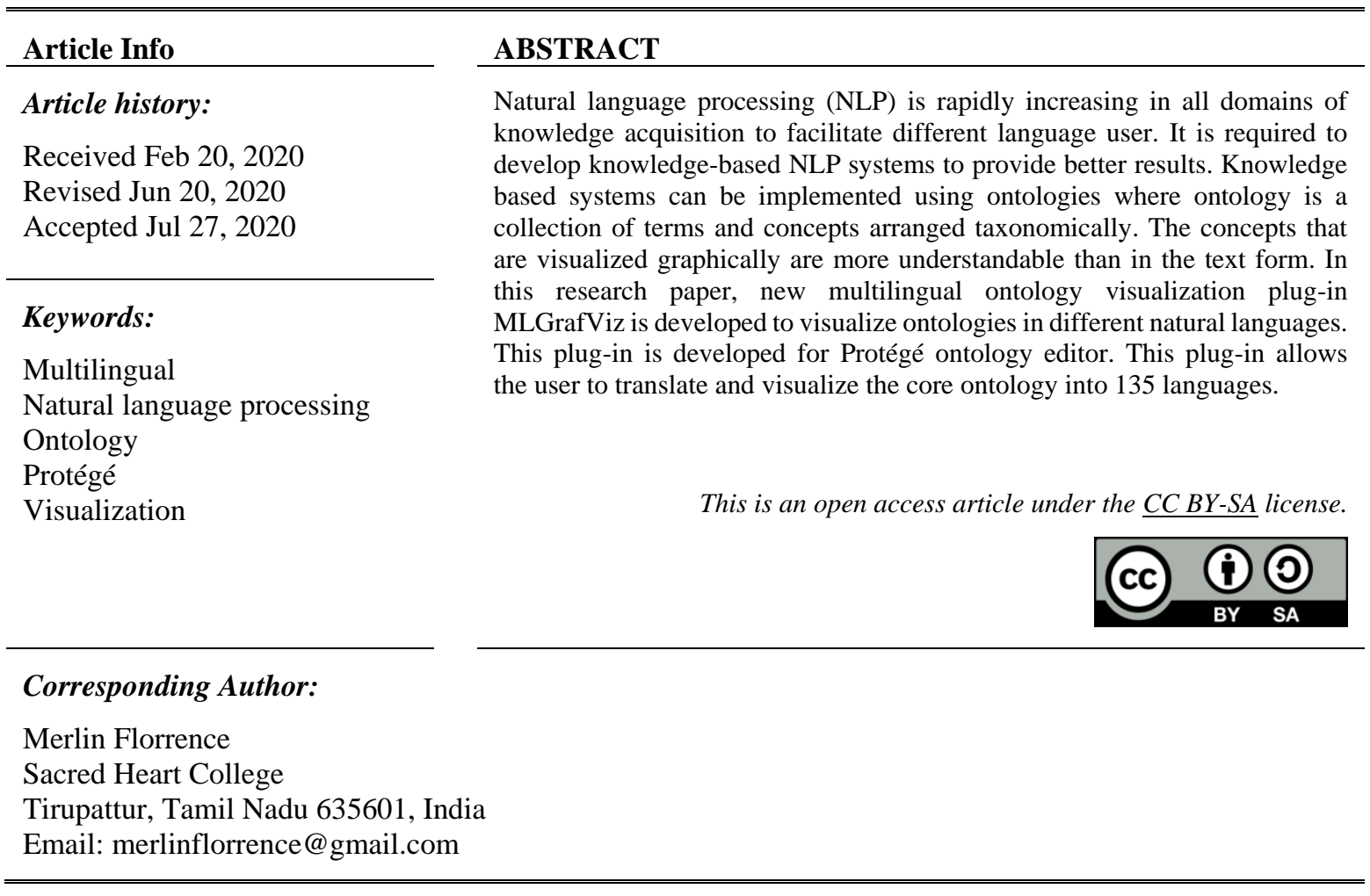

\section{INTRODUCTION}

Natural language processing (NLP) has been included in all domains of knowledge acquisition like education, business and many other technologies. People are accessing information through various sources like internet in their own language, to gain and share knowledge. At present, most of the devices like smart phones, tablets, smart televisions are developed with NLP technique to support the user to communicate in more than one language. The NLP is concerned with interacting machine and human languages. NLP implements knowledge based of information access for better results by adopting semantic web techniques [1]. Ontology plays an important role in building semantic networks. It constructs knowledge-based structure of the concepts. It comprises of collection of hierarchically arranged terms. Generally, it can be defined as, "the basic terms and relations comprising the vocabulary of a topic area as well as the rules for combining terms and relations to define extensions to the vocabulary" [2]. Numerous tools are developed to build ontologies like Protégé, OBOEdit, and SWOOP etc. Most of the ontology editors were built with plug-ins to perform the operations like evaluating, visualizing, querying and so on. Visualization is one of the important features which allows the user to visualize the developed ontology in the desired format. OntoGraf [3], OWLViz [4], Jambalaya [5] are some of the visualization plug-ins used to visualize and understand the structure of the ontology easily and effectively. Visualization plug-ins can be indented list, node-link and tree, zoomable, space-filling, focus with context or distortion, and 3D information landscapes [6]. Visualization tools represent the concepts and relationship between the concepts in different layout formats (radial, spring, horizontal tree, vertical tree, sphere, grid and symmetric). The aim of this paper is to build new ontology visualization plug-in to visualize ontology in different natural languages. The proposed plug-in MLGrafViz is developed to support 135 natural languages. The core ontology developed in any language can be translated into the user's desired 
language. The paper is organized as follows: the research work carried out on ontology visualization plug-ins used in Protégé ontology editor are analyzed in Section 2. The features of the proposed multilingual visualization plug-in are described in Section 3. Section 4 presents the performance analysis of the proposed plug-in. In Section 5, a comparative analysis of the proposed plug-in with the existing plug-ins is tabulated. Conclusions and future works are proposed in Section 6.

\section{STATE-OF-ART ON PROTÉGÉ ONTOLOGY VISUALIZATION PLUG-INS}

Ontology is a collection of hierarchically arranged terms. It can be represented graphically using many tools (OWLGrEd, NavigOWL etc.,) and plug-ins (Jambalaya, OntoGraf, GraphViz, GROW, OntoSphere [7], GrOWL [8] and so on). These tools and plug-ins are used to visualize the ontology primitives such as classes, data properties, object properties and instances effectively. Most of the visualization tools and plug-ins are developed with various visualization layouts (Tree, radial, spring, UML etc.) and filters. These tools use different shapes and symbols to represent the classes, cardinalities, and relations to distinguish them for an easy understanding [6]. It is identified that most of the ontology visualization tools are developed as a plug-in which can be integrated with the ontology editors like Protégé. The ontology visualization plug-ins used in Protégé are compared using common features and the ability to support natural languages. The results are tabulated in Table 1. The common features of the ontology visualization plug-ins used in Protégé are compared already with various parameters [9-13]. These reviews disclosed that the existing ontology visualizing plugins and tools are lacking in visualizing multilingual concepts. It is identified that very few tools like OWLGrEd are supported natural languages with some additional configurations. It is difficult to the user who wants to access and create ontology in own natural languages.

Table 1. Comparison of Protégé ontology visualization plug-ins

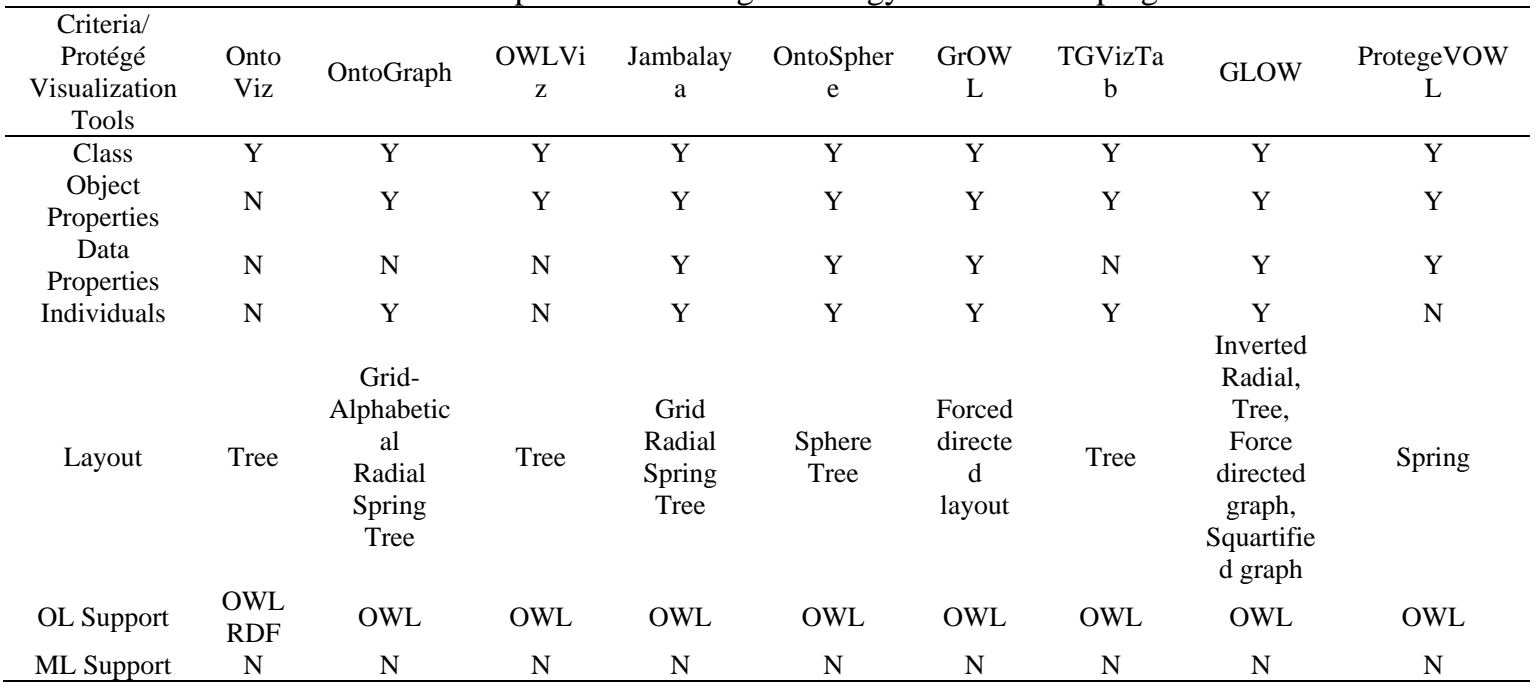

\section{ARCHITECTURE OF MLGRAFVIZ}

Multilingual ontologies are developed by adopting various ontology development methods like ontology mediation, ontology localization and ontology specification [14-19]. When visualizing the ontology using the tool like OntoGraf, it is imprecise in visualizing non-English languages. Sometimes the text is displayed as square boxes, though the rendering is applied. New ontology visualization plug-in MLGrafViz is developed as an extension of OntoGraf to overcome this defect. It is developed using dot, neato, fdp, sfdp, and twopi and circo layouts algorithms. The dot algorithm produces a ranked layout of a graph. The layout computed by neato is specified by a virtual physical model. The fdp layout is similar in appearance to neato and uses springs only between nodes connected with an edge. The sfdp layout is same as fdp but it uses a refined multilevel approach that enables to handle very large graphs. Twopi represents the radial layout of the graph. The circo is used to place connected components on a circle.

MLGrafViz is developed using Java and Graphviz algorithms. The architecture of this plug-in is given in Figure 1. Initially, it allows the user to create a new ontology or to import an existing ontology into Protégé workspace. The imported ontology will be displayed in a class browser. MLGrafViz enables the user to select the language to visualize the ontology. The request is submitted to Google translate API which performs 
statistical machine translation and then the terms are translated into the desired natural languages. Google translate API is an open source translator used to translate text, speech, images and videos from source language to target language. It provides an API which allows the developer to build an extension and software to translate the source. Google translate uses statistical analyses instead of rule-based analyses. Since ontology is hierarchically structured terms, statistical machine translator provides better result than the rule-based translator. Rule based machine translation is used in translating the passage grammatically. Finally, the translated terms are displayed in MLGrafViz panel.

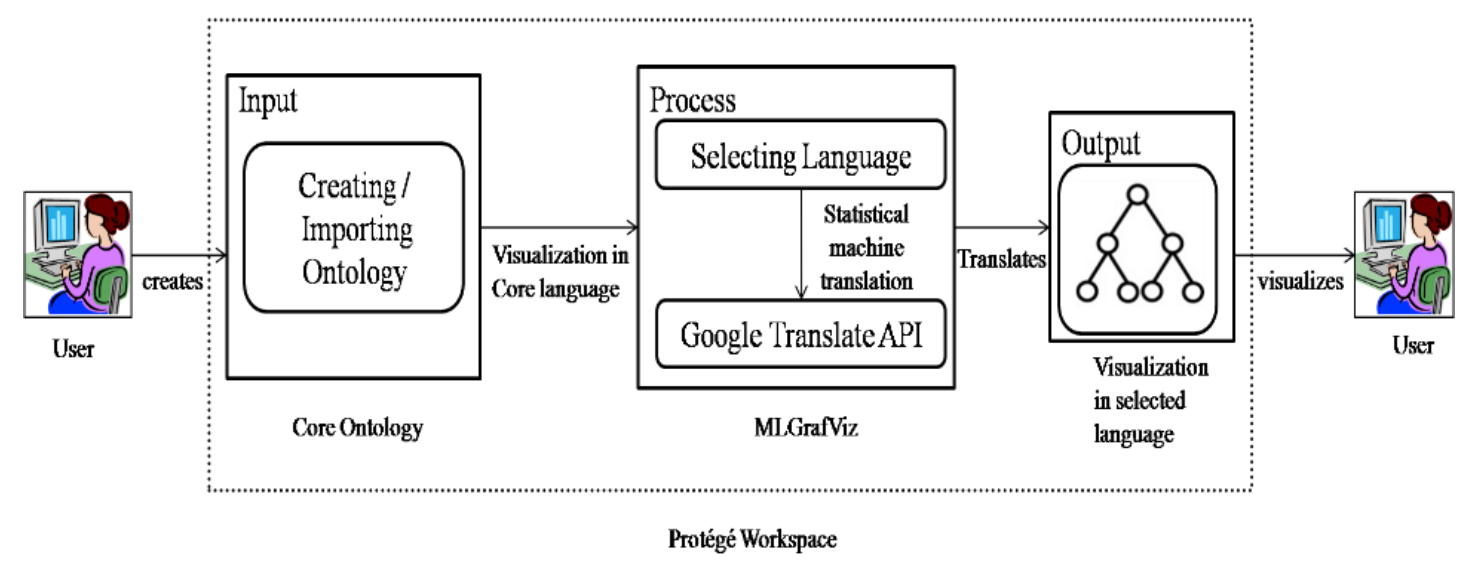

Figure 1. An architecture of MLGrafViz

MLGrafViz plug-in allows the user to visualize ontology into seven layouts: grid alphabetical, tree horizontal, tree vertical, spring, radial, directed horizontal and directed vertical. User can construct the core ontology in any natural language which will be translated and visualized into 135 different natural languages. The classes and individuals are represented in a rounded rectangle node. The relationships between the concepts and properties are represented as an Arc. This plug-in enables the user to save the graph as JPEG, Dot, and PNG and Graph format. It provides two types of tooltips for each node viz., class tooltip and individual tooltip. The class tooltip contains URI, annotations, equivalent classes, disjoint classes and superclasses and title of the label. Individual tooltips include URI, title of the node, annotations, data property assertions, different individuals, negative data property assertions, negative object property assertions, object property assertions and same individuals. User can perform three kinds of zooming viz., zoom-in, zoom-out and zoom-nil. MLGrafViz provides the following five methods of searching for the user: contains, starts with, ends with, exact match and regular expression. This plug-in also allows the user to import and export the graph. MLGrafViz requires constant internet connection to translate the source. Hyphenated words like Data_Type and Capitalized work like DataType are not able to translate.

\section{ANALYSIS OF MLGRAFVIZ}

\subsection{Testing with ontology application}

The ontology application for Computer Fundamentals is developed to validate the performance of the MLGrafViz plug-in. The core ontology for Computer Fundamentals is developed in English language. The objective of the proposed ontology for computer fundamentals is to help the beginners to understand the basics of computer. The concepts are arranged hierarchically. The descriptions of the concepts are expressed using annotation property. The relationships between the concepts are established using object property. The developed ontology is visualized using OntoGraf, OWLViz and ProtegeVOWL in core language. MLGrafViz facilitates the user to visualize the ontology in different natural languages without changing the core ontology structure as depicted in Figure 2 (a), (b), (c), (d). User can save the graph by using MLGrafViz for their further reference in different format like JPEG, DOT and Graph. The proposed plug-has the feature to export the graph in PNG format which can be used an input to OWLAX plug-in. OWLAX plug-in is used to build ontology using images by converting the images into ontologies. This plug-in allows the user to develop multilingual ontology applications without using ontology mediation techniques. The ontology developed for any domain can be visualized into user desired languages by using the proposed plug-in. 


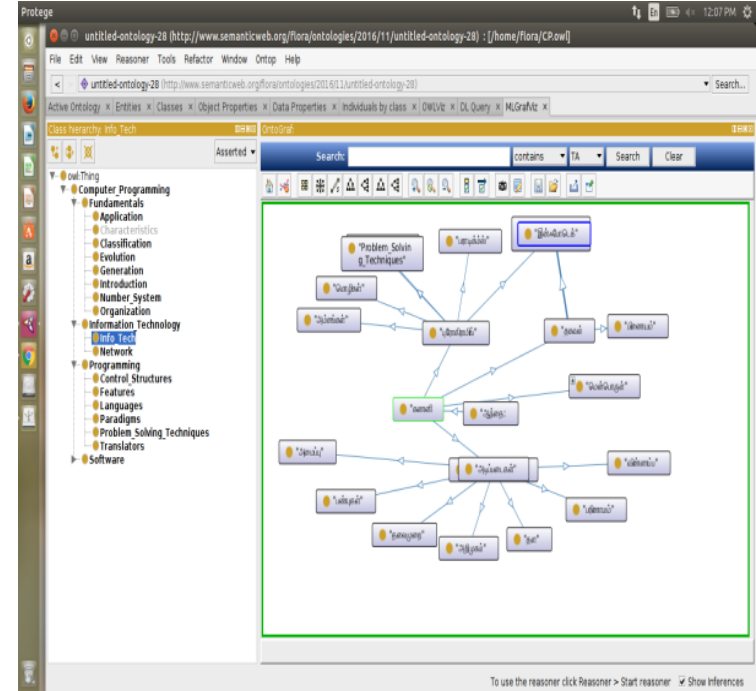

(a)Visualization in Tamil language

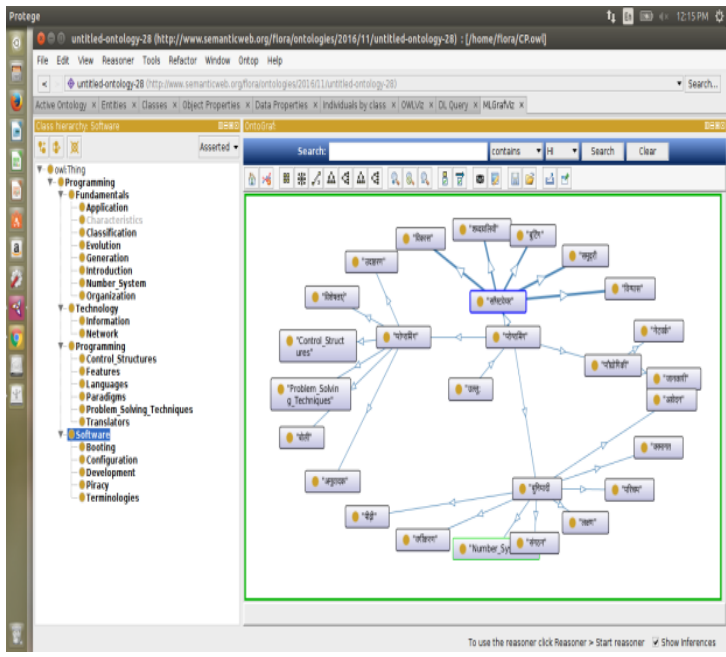

(c)Visualization in Hindi language

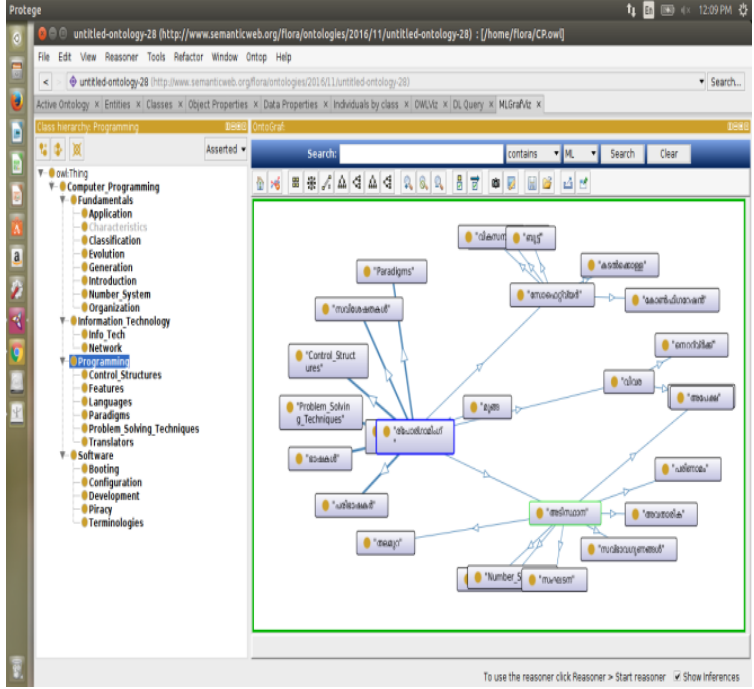

(b)Visualization in Malayalam language

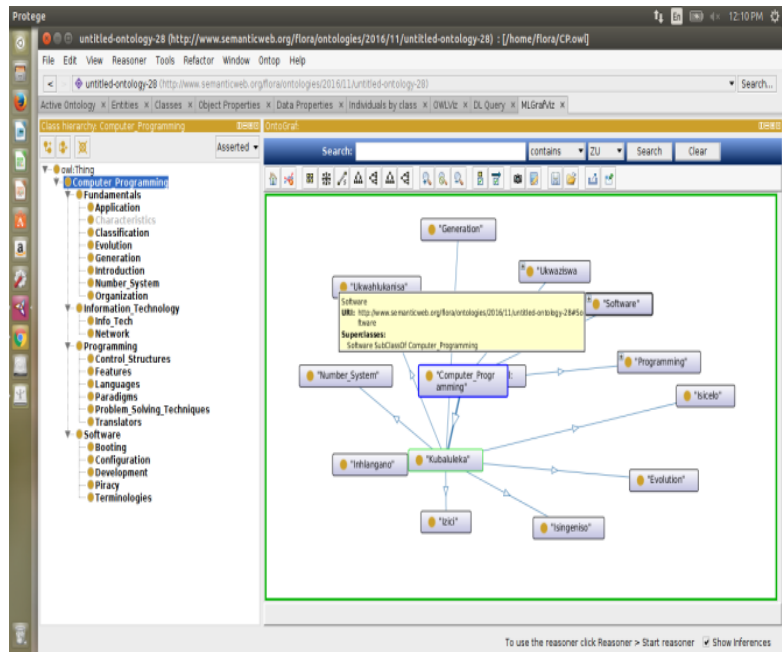

(d)Visualization in Zulu language

Figure 2. Visualization of computer fundamentals ontology in different natural languages

\subsection{Performance analysis of MLGrafViz}

MLGrafViz plug-in is compared with the other ontology visualization plug-ins used in Protégé 5.1.0 workspace with the following parameters: a) no. of. Characters accepted, b) no. of. Languages supported, c) size of panel, d) zoomable, e) dimension, f) technique used, g) layout, h) tool tips, i) import and j) export. The result of the comparison is given in Table 2. The results of the comparison study reveal that MLGrafViz gives better result in the perspective of multilingual visualization. Also, the proposed plug-in has bigger panel size than other visualization panel that enables the user to envision the ontology clearer. 
Table 2. Comparison of MLGrafViz with existing ontology visualization plug-ins

\begin{tabular}{|c|c|c|c|c|c|c|c|c|c|c|c|c|c|}
\hline $\begin{array}{l}\text { Ontology } \\
\text { Visualization } \\
\text { Tools }\end{array}$ & $\begin{array}{c}\text { No. of } \\
\text { Characters } \\
\text { accepted }\end{array}$ & $\begin{array}{c}\text { No. of. } \\
\text { Languages } \\
\text { Supported } \\
\end{array}$ & $\begin{array}{c}\text { Size of } \\
\text { Panel }\end{array}$ & Zoom & Dimension & $\begin{array}{c}\text { Technique } \\
\text { used }\end{array}$ & $\begin{array}{l}\text { Keyword } \\
\text { Search }\end{array}$ & Layout & Tool Tips & Import & Export & Annotations & Relation \\
\hline MLGrafViz & 100 & 135 & $800 \times 600$ & Yes & $2 \mathrm{D}$ & Zoomable & Yes & $\begin{array}{c}\text { Radial } \\
\text { Spring } \\
\text { Horizontal } \\
\text { Tree } \\
\text { Vertical } \\
\text { Tree } \\
\text { Radial }\end{array}$ & $\begin{array}{c}\text { Class } \\
\text { Tooltip } \\
\text { Individual } \\
\text { Tooltip }\end{array}$ & Yes & Yes & Yes & $\begin{array}{c}\text { has } \\
\text { subclass }\end{array}$ \\
\hline OntoGraf & 75 & 1 & $800 \times 300$ & Yes & $2 \mathrm{D}$ & Zoomable & Yes & $\begin{array}{c}\text { Spring } \\
\text { Horizontal } \\
\text { Tree } \\
\text { Vertical } \\
\text { Tree }\end{array}$ & $\begin{array}{c}\text { Class } \\
\text { Tooltip } \\
\text { Individual } \\
\text { Tooltip }\end{array}$ & Yes & Yes & Yes & $\begin{array}{c}\text { has } \\
\text { subclass }\end{array}$ \\
\hline OWLViz & 75 & 1 & $800 \times 300$ & Yes & $2 \mathrm{D}$ & $\begin{array}{c}\text { Node Link } \\
\text { and Tree }\end{array}$ & No & $\begin{array}{c}\text { Horizontal } \\
\text { Tree }\end{array}$ & No & No & Yes & No & is-a \\
\hline $\begin{array}{l}\text { SuperClass } \\
\text { Hierarchy }\end{array}$ & 75 & 1 & $800 \times 300$ & No & $2 \mathrm{D}$ & $\begin{array}{l}\text { Indented } \\
\text { List }\end{array}$ & No & $\begin{array}{l}\text { Indented } \\
\text { Hierarchy }\end{array}$ & No & No & No & No & No \\
\hline
\end{tabular}

\section{CONCLUSION}

The paper presents the MLGrafViz plug-in for visualizing ontologies in different natural languages. The tool is developed to facilitate different language users to understand the content in their own language. This plug-in is developed for Protégé 5.1.0 workspace and it needs Internet connection to translate the terms. It uses Google translate API for translating the sources. In future, the standalone application like OWLGrEd can be developed to visualize ontologies in different natural languages. Rule based machine translation can be added with this plug-in to give grammatically translated result. It is also possible to build a multilingual visualization plug-in that can be integrated with other ontology editors than Protégé.

\section{REFERENCES}

[1] Busch, J. E., Lin, A. D., Graydon, P. J., \& Caudill, M. U.S. Patent No. 7,027,974. Washington, DC: U.S. Patent and Trademark Office, 2006.

[2] Gomez-Perez, A., Fernández-López, M., \& Corcho, O. "Ontological Engineering: with examples from the areas of Knowledge Management, e-Commerce and the Semantic Web.” Springer Science \& Business Media, 2006.

[3] Falconer, Sean. OntoGraf. ProtegeWiki. [Online] April 12, 2010. [Cited: January 18, 2013.] http://protegewiki.stanford.edu/index.php/OntoGraf.

[4] Horridge, Matthew. OwlViz. ProtegeWiki. [Online] March 4, 2010. [Cited: July 23, 2013.] http://protegewiki.stanford.edu/wiki/Special:FormEdit/Plugin/OWLViz.

[5] Lintern, R., \& Storey, M. A. "Jambalaya Express: Ontology visualization-on-demand." In International Protégé Conference, 2005.

[6] Katifori, Akrivi, Constantin Halatsis, Giorgos Lepouras, Costas Vassilakis and Eugenia G. Giannopoulou. "Ontology visualization methods - a survey." ACM Comput. Surv, vol. 39, p. 10, 2007.

[7] Bosca, Alessio, Dario Bonino and Paolo Pellegrino. "OntoSphere: more than a 3D ontology visualization tool." SWAP, 2005.

[8] Krivov, Sergey \& Williams, Richard \& Villa, Ferdinando. "GrOWL: A tool for visualization and editing of OWL ontologies.” Web Semantics: Science, Services and Agents on the World Wide Web. vol. 5, no. 2, pp. 54-57, 2007.

[9] T. Boinski, A. Jaworska, R. Kleczkowski and P. Kunowski. "Ontology visualization.” 2010 2nd International Conference on Information Technology, (2010 ICIT), Gdansk, pp. 17-20, 2010.

[10] Swaminathan, Venkataraman and R. Sivakumar. "A Comparative Study of Recent Ontology Visualization Tools with a Case of Diabetes Data." International Journal of Research, vol. 2, no. 3, pp. 31-36, 2012.

[11] Sivakumar, R., Arivoli, P. V., \& Sri, A. V. V. M. "Ontology visualization PROTÉGÉ tools-a review." International Journal of Advanced Information Technology (IJAIT), Vol. 1, No. 4, pp. 1-11, August 2011.

[12] Lourdusamy, Ravi, Arulnathan, Vikkiramadhithan and Ganapathy Gopinath. "A Survey on Ontology Visualizations." National Conference on Software Engineering and Applications, 2008.

[13] Lourdusamy, Ravi \& Florrence, Merlin. (2016). Methods, Approaches, Principles, Guidelines and Applications on Multilingual Ontologies: A Survey. ICTACT Journal on Soft Computing. vol. 7, no. 1, pp. 1350-1358 - October 2016.

[14] R. Lourdusamy and M. F. Joseph, "A multilingual ontology for Tamil Literary works," 2016 3rd International Conference on Advanced Computing and Communication Systems (ICACCS), Coimbatore, pp. 1-42016.

[15] Alani, Harith. "TGVizTab: An Ontology Visualisation Extension for Protégé." Knowledge Capture (K-Cap'03), Workshop on Visualization Information in Knowledge Engineering, 2003. 
[16] Hop, W., Ridder, S. d., \& Frederik Hogenboom and Flavius Frasincar. (n.d.). "GLOW is a visualization for OWL ontologies, based on Hierarchical Edge Bundles.” from GlowVis: https://www.glowvis.org/Main_Page, Retrieved 102015 ,

[17] Lohmann, Steffen, Negru, Stefan \& Haag, Florian, Ertl, Thomas. "Visualizing ontologies with VOWL." Semantic Web. vol. 7, no. 4, pp. 399-419, May 2016.

[18] Sintek, Michael. OntoViz. ProtegeWiki. [Online] July 11, 2007. [Cited: August 31, 2010.] http://protegewiki.stanford.edu/index.php/OntoViz. 